\title{
CONFORMIDADE CONTÁBIL-FISCAL E GERENCIAMENTO DE RESULTADOS CONTÁBEIS NO
} BRASIL

\author{
Book-Tax conformity and earnings management in Brazil
}

Antonio Lopo Martinez

E-mail: antoniolopomartinez@gmail.com

Doutor em Contabilidade pela Universidade de São Paulo; Mestre em Business Administration na University of California, Berkeley; Doutorando em Direito na Universidade de Coimbra/Universidad de Salamanca; Pesquisador Convidado no Max Planck Institute of Tax Law and Public Finance. Endereço para contato: Marstallpl. 1, Room M 413, 80539, München, Deutschland. http://orcid.org/0000-0001-9624-7646

Lennilton Viana Leal

E-mail: lenniltonleal@gmail.com

Mestre em Contabilidade pela Fundação Instituto Capixaba de Pesquisa em Contabilidade, Economia e Finanças (Fucape Business School); Especialista em Auditoria Contábil e Financeira pelo Centro de Ensino Superior Vale do Parnaíba (Cesvale); Doutorando em Ciências Contábeis pela Fundação Instituto Capixaba de Pesquisa em Contabilidade, Economia e Finanças (Fucape

Business School).

http://orcid.org/0000-0001-9220-7479 


\section{Resumo}

Este estudo se propôs a examinar se uma maior conformidade contábil-fiscal restringe o gerenciamento de resultados em companhias de capital aberto, não financeiras, listadas na B3 no período de 2010 a 2016. A conformidade contábil-fiscal mensura o grau de aderência das normas da contabilidade societária e daquelas da contabilidade tributária. Metodologicamente, foram utilizados três modelos de regressões, em que os dois primeiros modelos têm o objetivo de fornecer, respectivamente, o grau de conformidade contábil-fiscal e os accruals discricionários, que serão utilizados no terceiro modelo, o qual é o foco principal de análise, em que se investigam associação entre a conformidade contábil-fiscal e o gerenciamento de resultados contábeis. Os resultados mostraram que quanto menor a conformidade contábilfiscal, maior é a propensão ao gerenciamento de resultados. Adicionalmente, foi identificado que as empresas de menor tamanho e com menor rentabilidade tendem a gerenciar mais seus resultados contábeis. Os achados indicam que práticas contábeis que reduzem a conformidade das regras da contabilidade societária com aquelas da contabilidade tributária estimulam aumento do gerenciamento de resultados contábeis. Por sua vez, naquelas empresas com maior conformidade contábil-fiscal há uma menor propensão a gerenciamento de resultados contábeis. O grau de conformidade contábil-fiscal passa a ser, assim, um elemento determinante para compreender os incentivos econômicos ao gerenciamento de resultados contábeis no Brasil. Face as conclusões expostas, esta pesquisa é de significativo interesse para reguladores, profissionais e usuários da contabilidade, que podem identificar possíveis efeitos na qualidade da informação contábil de uma menor conformidade contábil-fiscal.

Palavras-chave: Conformidade contábil-fiscal. Gerenciamento de resultados. Accruals discricionários.

\section{Abstract}

This study aimed to examine whether higher Book-Tax conformity restricts earnings management in public companies, non-financial corporations, listed in B3 in the period from 2010 to 2016. Book-Tax conformity measures the degree of compliance of the corporate accounting and those of tax accounting. Methodologically, three regression models were used, in which the first two models aim to provide, respectively, the degree of Book-Tax conformity and discretionary accruals, which will be used in the third model, which is the main focus of analysis, which investigates the association between Book-Tax conformity and earnings management. The results showed that the lower the Book-Tax conformity, the higher the propensity to manage results. Also, it was identified that smaller companies with lower profitability tend to manage their accounting results more. The findings indicate that accounting practices that reduce the conformity of corporate accounting rules with those of tax accounting encourage increased earnings management. On the other hand, in those companies with higher book-tax compliance, there is a lower propensity to manage accounting results. The degree of Book-Tax conformity becomes, therefore, a determinant element to understand the economic incentives to earnings management in Brazil. Given the above conclusions, this research is of significant interest to regulators, professionals, and users of accounting, who can identify possible effects on the quality of accounting information from lower Book-Tax conformity.

Keywords: Book-Tax conformity. Earnings management. Discretionary accruals. 


\section{INTRODUÇÃO}

O trabalho tem como foco o estudo de empresas brasileiras de capital aberto, listadas na B3 - Brasil, Bolsa, Balcão, e a verificação do exame da conformidade Book-Tax em observar se podem restringir a divulgação, pelos gestores, de resultados contábeis e tributários. De acordo com exposição de Graham, Raedy, e Shackelford (2012), a diferença entre o lucro contábil e o lucro tributável, denominada Book-Tax Difference (BTD), apresenta-se como um tema complexo e atual. $O$ estudo BTD, de acordo com Hanlon e Heitzman (2010), tem sido considerado uma área de investigação de relevância no campo tributário.

De acordo com Formigoni, Antunes, e Paulo (2009, p. 46), o BTD surge em razão: das divergências entre as regras de contabilidade e as regras fiscais (Diferenças Book-Tax normais ou não discricionárias) e da gestão de resultados (Diferenças Book-Tax anormais ou discricionárias).

De acordo com Martinez (2008), gerenciar resultados consiste na manipulação das informações financeiras pelos administradores. Burgstaler, Leuz, e Hail (2006) revelam que o desempenho de uma empresa não pode ser mensurado por meio do lucro. Segundo os autores, os administradores escolhem critérios contábeis para gerenciar os resultados, de forma oportuna, diminuindo a qualidade do lucro reportado.

A conformidade contábil-fiscal (Book-Tax Conformity) é definida como o grau de aderência do lucro tributável ao lucro antes dos tributos - LAIR (Atwood, Drake, \& Myers, 2010). De acordo com Desai (2005), uma alta conformidade contábil-fiscal reduz o gerenciamento fiscal, aumenta a eficiência da informação contábil reportada e o cumprimento das normas fiscais.

Os oponentes argumentam que uma alta conformidade contábil-fiscal restringe a flexibilidade dos relatórios, afetando de forma adversa a divulgação dos demonstrativos financeiros fornecidos aos mercados de capitais (Hanlon, Maydew, \& Shevlin, 2008; Hanlon \& Shevlin, 2005).

A presente pesquisa se justifica no Brasil face às mudanças ocorridas na legislação societária, as Leis n. 11.638/07 (2007) e n. 11.941/09 (2009), que proporcionaram uma maior flexibilidade para reconhecer, mensurar e evidenciar a informação contábil, e a uma diminuição do fator tributário nas questões societárias, reduzindo a conformidade contábilfiscal (Ferreira, Martinez, Costa, \& Passamani, 2012).

Diante disso, este estudo contribui ao examinar o efeito da conformidade contábilfiscal na qualidade dos lucros divulgados, associando a gestão de resultados contábeis e - planejamento tributário das companhias brasileiras de capital aberto, listadas na B3 Brasil, Bolsa, Balcão. Em suma, almeja-se conhecer como variações no grau de conformidade 
contábil-fiscal impactam a propensão ao gerenciamento de resultados. Havendo relação, esta é direta ou inversa?

A resposta a essa pergunta auxilia os diversos interessados na informação contábil a estimar, avaliar e mensurar a qualidade dos demonstrativos financeiros. Em uma eventual redução da conformidade contábil-fiscal, o que se deve esperar em termos incentivos à prática de earnings management.

Adverte-se que embora o tema BTD seja explorado na literatura corrente, a discussão do efeito do grau de conformidade contábil e fiscal não foi ainda, salvo melhor juízo, feita em nível de Brasil, sendo, portanto, um tema de pesquisa em aberto e em uma plataforma especial que o contexto brasileiro proporciona. Ainda nesse ponto não se deve confundir o BTD diretamente com a conformidade fiscal. Na realidade são conceitos que têm a mesma origem da diferença entre as regras contábeis e fiscais, mas que, contudo, o primeiro focase na diferença em termos quantitativos do lucro contabil e fiscal, e, o segundo, aprecia o inverso que é justamente quando esses resultados convergem e quais os efeitos na qualidade dos lucros.

O presente trabalho utiliza, para a sua modelagem, uma amostra de 367 empresas brasileiras de capital aberto, listadas na B3 - Brasil, Bolsa, Balcão, no período de 2010 a 2016. Todos os dados foram coletados no Economática e foram excluídas da amostra as instituições financeiras, pois suas demonstrações financeiras possuem características e são elaboradas por práticas contábeis diferenciadas, que podem dificultar a análise. Os modelos a serem implementados foram testados a partir da ferramenta de Análise de Regressão Múltipla (MQO). São utilizados três modelos de regressões, em que um conjunto de variáveis é utilizado para explicar a influência que possuem sobre as variáveis de interesse.

Nesse sentido, o artigo se apresenta organizado em seções, sendo a primeira a introdução, com exposição de objetivo, justificativa e contribuições. Em seguida o texto apresenta a segunda seção, na qual se expõem os conceitos de Box-Tax Difference, gerenciamento de resultados e a conformidade contábil-fiscal. Após, apresenta-se a metodologia aplicada para o estudo na terceira seção; na quarta seção são mostrados os resultados das análises desenvolvidas e, ao final, as conclusões da pesquisa.

\section{REFERENCIAL TEÓRICO}

\subsection{DIFERENÇAS ENTRE LUCRO CONTÁBIL E LUCRO TRIBUTÁRIO}

O BTD é a divergência entre o lucro contábil e o lucro tributável e é oriundo de alguns fatores (Hanlon \& Heitzman, 2010)). Esses fatores decorrem: das divergências entre 
as normas contábeis, que têm por objetivo o registro dos fatos econômicos ocorridos e a diminuição da assimetria informacional; e das normas tributárias, que têm por objetivo os cálculos dos tributos, obedecendo à legislação tributária.

As BTDs são divididas em duas categorias: permanentes, decorrentes da manipulação dos administradores e planejamento tributário, evidenciando previsões futuras nas despesas com tributos; e temporárias, decorrentes dos ajustes no reconhecimento das receitas e das despesas, em períodos diferentes, resultantes do regime de competência e de regime de caixa (Ferreira et al., 2012).

Riguen e Jarboui (2017) revelam que as BTDs ajudam a fornecer informações sobre o nível da qualidade dos ganhos, enquanto um aumento na conformidade Book-Tax ajuda a melhorar a qualidade da informação.

A literatura contábil documenta que os estudos sobre BTD são uma área importantes na análise da qualidade dos lucros. Para fins de elaboração de relatórios, contribuem com informações úteis para investidores, credores e outros usuários da informação contábil a tomarem as melhores e mais eficazes decisões (Hanlon, 2005; Lev \& Nissim, 2004; Blaylock, Shevlin, \& Wilson, 2012; Wahab \& Holland, 2015).

Guenther (2001) sugere que as BTDs são significativas, sendo as diferenças transitórias entre as regras contábeis e fiscais, e estão associadas a ganhos futuros com informações incrementais para os usuários das demonstrações financeiras. As BTDs estão associadas a ganhos futuros, cujo foco se direciona para a captação de atividades de gerenciamento de resultados, conforme exposição dos estudos de Lev e Nissim (2004) e Hanlon (2005).

Dessa forma, os gestores podem manipular para cima o lucro contábil e para baixo - lucro fiscal, sem que uma decisão afete diretamente a outra. O estudo sobre as diferenças Book-Tax pode ser visto como uma proxy da manipulação de resultados, concentrando-se na divulgação oportunista dos gestores ao relatar os resultados contábeis, fiscais e a qualidade do lucro (Ferreira et al., 2012). Pesquisas anteriores, como as de Tang e Firth (2012), investigam se o BTD está associado à gestão de resultado e ao planejamento tributário. As pesquisas mostraram que gerenciar resultados explica parte do BTD e que este tem um efeito tributário mais poderoso do que o efeito no lucro dos relatórios contábeis.

\subsection{GERENCIAMENTO DE RESULTADOS CONTÁBEIS}

As demonstrações financeiras e demais relatórios contábeis são os principais meios de divulgação do desempenho financeiro e de controle das empresas (Peek, Palepu, \& Healy, 2010). Martinez (2013) define gerenciamento de resultados como o processo de decisões impostas pelas regras contábeis para apresentar o nível de resultado desejado. 
gerenciamento de resultado pode influenciar a política tributária da empresa. A hipótese de uma maior discrição no cálculo da renda financeira, quando comparada com os rendimentos tributáveis, permitirá que os administradores utilizem critérios para gerir de forma positiva a renda, sem aumentar o lucro tributável (Phillips, Pincus, \& Rego, 2003). Leuz, Nanda, e Wysocki (2003) definem gestão de resultados como a tentativa usada pelos administradores da empresa em proteger suas posições e benefícios, manipulando informações financeiras.

Segundo a análise realizada por Roychowdhury (2006), observando ações de administradores que se desviam de boas práticas contábeis, a gestão de resultados tem o foco de divulgar os resultados em contextos que atendam à necessidade desses administradores.

Assim, importante entender que de acordo com exposição de Martinez (2013), o gerenciamento de resultados não deve ser visto como fraude contábil, uma vez que os gestores têm opção em utilizar procedimentos para escolhas contábeis, mesmo que estas não reflitam a realidade da empresa, mas exposição de resultados que o gestor quer.

Nesse sentido, a prática de manipulação de informações contábeis pode ser realizada de três formas, sendo a primeira por meio do gerenciamento de resultados em aplicação de accruals, a segunda forma decorre da manipulação e classificação de elementos e demonstrativos contábeis, e a terceira decorre da manipulação de atividades operacionais, segundo explicam Paulo, Martins, e Corrar (2007).

Segundo Comprix, Graham, e Moore (2011), o gerenciamento de resultados e o gerenciamento tributário podem ser revelados com base nas análises dos conteúdos dos tipos de BTD. As consequências das práticas oportunistas dos gestores podem levar ao comprometimento financeiro das empresas e dos resultados dos exercícios e à confiança dos investidores na empresa (Hanlon \& Shevlin, 2005).

\subsection{CONFORMIDADE CONTÁBIL-FISCAL}

A divulgação de demonstrações financeiras e a elaboração destas se vinculam com a conformidade Book-Tax em finalidade de elaboração dos mesmos fins fiscais, segundo explicam Hanlon et al. (2008), em conclusão que o nível de conformidade Book-Tax se apresenta forte, quando ocorre o papel informacional de lucros expressos pelos demonstrativos financeiros em redução.

Dessa forma, conforme explica Desai (2005), a Conformity Book-Tax pode estimular as empresas a reduzirem o planejamento tributário abusivo em face de serem menos agressivas em divulgação de lucros para o mercado de capitais. Essa atuação explicita que 
a conformidade Book tax propicia certa disciplina para os administradores em realizarem discricionariedade em aumento de qualidade da divulgação financeira da instituição.

Chan, Lin, e Tang (2013) esclarece que as empresas ao estarem inseridas em ambientes com alto nível de conformidade, elas se deparam com um trade-off financeiro-fiscal, em que as decisões para fins contábeis afetam, diretamente, as decisões para fins fiscais, limitando as firmas, tornando mais custosos para agirem oportunisticamente.

Blaylock, Gaertner, \& Shevlin (2015) destacam duas possíveis razões para justificar a relação positiva entre altos níveis de conformidade Book-Tax e gerenciamentos de resultados: alinhar as medidas de lucro contábil e fiscal reduz as habilidades dos investidores de perceberem gerenciamento de resultados; e as firmas podem suavizar seus lucros por meio de financiamento de dívidas.

Os opositores de um elevado nível de conformidade Book-Tax argumentam que a informação fornecida aos usuários dos demonstrativos financeiros é diferente das que são requeridas pelo Fisco, a conformidade Book-Tax se traduz em uma redução da qualidade dos lucros divulgados (Hanlon \& Shevlin, 2005; Hanlon et al., 2008; Atwood et al., 2010).

Hanlon e Shevlin (2005) afirmam que uma alta conformidade Book-Tax pode causar perda da informação para o mercado de capitais, pois tanto o lucro contábil quanto o lucro tributável fornecem informações essenciais, e, se essas medidas forem modificadas, haverá perda na qualidade da informação para os investidores. Atwood et al. (2010) argumentam que uma alta conformidade Book-Tax pode restringir os gestores de usarem sua discricionariedade para divulgar lucros oportunisticamente, porém, a qualidade do lucro é menor.

Diante da fundamentação teórica e do objetivo proposto neste trabalho, formula-se a seguinte hipótese de pesquisa:

H: Quanto maior a conformidade contábil-fiscal, menor o gerenciamento de resultados contábeis.

A seguir serão apresentados os procedimentos metodológicos da pesquisa.

\section{METODOLOGIA DA PESQUISA}

A metodologia utilizada para testar a hipótese foi adaptada dos trabalhos de Atwood et al. (2010) e Tang (2014), que realizaram investigações semelhantes. Este estudo se trata de pesquisa empírica com o uso de técnicas de análise estatística multivariada, com o auxílio do software STATA. Os modelos a serem implementados foram testados a partir da ferramenta de Análise de Regressão Múltipla com dados empilhados (pooled). 


\subsection{SELEÇÃO DA AMOSTRA}

A coleta de dados foi baseada em uma amostra constituída pelas empresas brasileiras de capital aberto, listadas na B3 - Brasil, Bolsa, Balcão, no período compreendido entre 2010 e 2016. Essa delimitação do período e a opção por não incluir os anos anteriores a 2010 se devem ao fato de que, no Brasil, as normas para elaboração dos demonstrativos financeiros com finalidades gerenciais e fiscais eram muito vinculadas, e as exigências do fisco afetavam as decisões dos gestores nas escolhas de critérios para a elaboração deles.

Assim, após as reestruturações do modelo contábil aos padrões internacionais com a adoção das normas do Internacional Financial Reporting Standards (IFRS) foram eliminadas as influências do fisco sobre a elaboração dos demonstrativos financeiros que geraram uma maior relação entre as normas contábeis e fiscais. Limitou-se ao ano 2016, por ser este o período mais recente. Os dados referentes à amostra foram obtidos por meio da base de dados do software Economática ${ }^{\circledR}$. De uma amostra original composta por 402 empresas ativas na B3, após a exclusão das instituições financeiras em razão das particularidades do setor, por possuírem normas contábeis e tributárias diferentes das demais empresas da amostra, a quantidade foi reduzida a 367 empresas que, multiplicadas pelo período de análise, totalizam 2.452 observações.

Tabela 1

Composição da amostra

\begin{tabular}{lr}
\hline \multicolumn{1}{c}{ Descrição } & Observações \\
\hline Empresas brasileiras de capital aberto ativas na B3 & 402 \\
(-) Exclusão de empresas do setor financeiro e seguro & $(35)$ \\
(=) Quantidade de empresas investigadas & 367 \\
(x) Quantidade de anos investigados & 7 \\
(=) Quantidade de observações (empresa/ano) & 2.569 \\
(-) Empresas/ano sem informações & $(117)$ \\
(=) Quantidade de observações utilizadas & 2.452 \\
\hline
\end{tabular}

\subsection{MODELO 1 - REGRESSÃO DA CONFORMIDADE CONTÁBIL- FISCAL}

Atwood et al. (2010) definem conformidade Book-Tax como a liberdade que a instituição tem para divulgar o lucro tributável e o lucro antes dos tributos - LAIR. Segundo os autores, os gestores têm incentivos para divulgar melhores resultados financeiros para os investidores e diminuem a parcela do imposto a pagar. 
Atwood et al. (2010) utilizam o modelo econométrico a seguir para analisar a conformidade Book-Tax:

$$
C T E_{i, t}=\beta o+\beta 1 P T B I_{i t}+\beta 2 D I V_{i, t}+\varepsilon_{i t}
$$

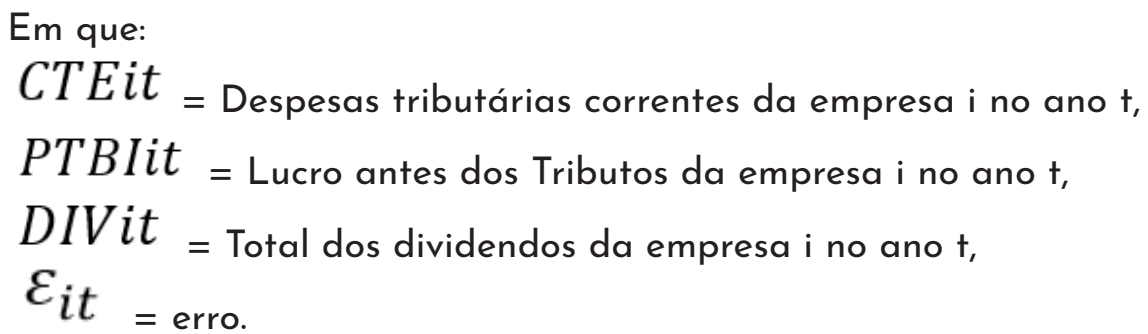

No modelo original existia a variável "ForPTBI", que corresponde ao lucro das empresas no exterior, estimado antes dos tributos. Como na base de dados do software Economática ${ }^{\circledR}$ não são disponibilizados os dados da despesa corrente tributária das empresas no exterior para medir a variável "ForPTBI", ela foi excluída do modelo utilizado neste trabalho. Atwood et al. (2010) fizeram testes no modelo sem a presença da variável "ForPTBI" e os resultados foram semelhantes com a presença da variável "ForPTBI".

Nesse modelo, o objetivo implica analisar se as variáveis e influenciam os resultados nas despesas tributárias correntes da empresa. Observar que no contexto brasileiro os juros sobre o capital próprio são considerados dividendos e se constituem em despesas dedutíveis tributariamente. Desse modo, a proposta de Atwood et al. (2010) é perfeitamente compatível na realidade brasileira.

\subsection{MODELO 2 - REGRESSÃO DO GERENCIAMENTO DE RESULTADOS CONTÁBEIS}

Segundo Dechow e Dichev (2002), os accruals são ajustes temporários na elaboração do fluxo de caixa das empresas, função do regime de competência. Os autores separam os accruals totais em: accruals discricionários associados ao comportamento oportunista e accruals não discricionários associados ao nível de atividades da empresa. Accruals discricionários são usados para mensurar a gestão de resultados e, quanto maior os accruals, mais forte e mais evidente o ato de gerenciar resultados (Goulart, 2007; Martinez, 2013).

Assim, para a devida medida correta dos accruals discricionários é relevante a avaliação dos accruals totais, sendo aplicadas as informações extraídas do Balanço Patrimonial, bem 
como da Demonstração do Resultado do exercício e demonstrativo do fluxo de caixa, em acordo com exposição de Goulart (2007), Martinez (2013) e Paulo (2007).

Neste trabalho, para se obterem os valores dos accruals totais se utilizou o método da abordagem de balanço, conforme a Equação 2:

$$
T A C C_{i t}=\left[\Delta A C_{i t}+\Delta C E C_{i t}-\Delta P C_{i t}-\Delta P a g_{C} P_{i t}-\left(\text { Depre }_{i t}+\text { Amort }_{i t}\right)\right] / A_{i, t-1}
$$

Em que:

$\operatorname{TACC}_{i t}=$ Total de accruals da empresa i no ano $\mathrm{t}$,

$\triangle A C_{i t}=\mathrm{A}$ diferença entre o ativo circulante da empresa i no ano te (t-1),

$\triangle C E C_{i t}=\mathrm{A}$ diferença entre caixas e equivalente à caixa da empresa $\mathrm{i}$ no ano $\mathrm{t}$,

$\Delta P C_{i t}=\mathrm{A}$ diferença entre o passivo circulante da empresa $\mathrm{i}$ no ano $\mathrm{t}$ e $(\mathrm{t}-\mathrm{l})$,

$\Delta \mathrm{Pag}_{C} P_{i t}=\mathrm{A}$ diferença entre os pagamentos de curto prazo i no ano te $(\mathrm{t}-1)$,

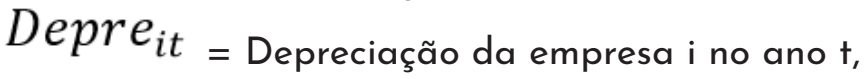

Amort $_{\text {it }}=$ Amortização da empresa i no ano $t$

$A_{i, t-1}=$ Ativos Totais da empresa i no ano (t-1).

O modelo econométrico utilizado para analisar o gerenciamento de resultado será o modelo proposto por Dechow, Sloan, e Sweney (1995), conhecido como Modelo Jones Modificado e Kothari, Leone, e Wasley (2005). Segundo Martinez (2013), esse modelo de apuração de accruals discricionários, ainda que não esteja à prova de críticas, sua simplicidade e funcionalidade tornaram-no o mais utilizado da literatura para mensurar os accruals discricionários.

$T A C C_{\mathrm{i}, \mathrm{t}}=\alpha_{1}\left(\frac{1}{A_{i, t-1}}\right)+\alpha_{2}\left(\Delta \Re V_{i t}-\Delta \Re C_{i t}\right)+\alpha_{3} P P E_{i t}+\alpha_{4} R O A_{i, t}+\varepsilon_{i t}$

Em que:

$T A C C_{\mathrm{i}, \mathrm{t}}=$ Accruals não discricionários da empresa i no ano $\mathrm{t}$,

$A_{i, t-1}=$ Ativos Totais da empresa i no ano ( $\left.t-1\right)$,

$\Delta \mathfrak{R} V_{i t}=\mathrm{A}$ diferença entre receita da empresa $\mathrm{i}$ no ano $\mathrm{t}$ e $(\mathrm{t}-\mathrm{l})$ dividida por $A_{i, t-1}$,

$\Delta \mathfrak{R} C_{i t}=\mathrm{A}$ diferença entre as contas a receber firma $\mathrm{i}$, ano $\mathrm{t}$ e $(\mathrm{t}-1)$ dividida por $A_{i, t-1}$

$P P E_{i t}=$ Ativo imobilizado da empresa $\mathrm{i}$ no ano $\mathrm{t}$ dividido por $A_{i, t-1}$, 
$\mathrm{ROA}_{\mathrm{i}, \mathrm{t}}=$ Retorno sobre Ativos da empresa i no ano + dividido por $A_{i, t-1}$,

$\mathcal{E}_{\text {it }}$ Accrual anormal empresa i no ano t.

Para o segundo modelo, o objetivo é avaliar se o ativo total, a variação da receita, a variação das contas a receber, o ativo imobilizado e intangível e o retorno dos ativos influenciam os resultados dos accruals totais.

\subsection{MODELO 3 - REGRESSÃO DO TESTE DA RELAÇÃO ENTRE A CONFORMIDADE CONTÁBIL-FISCAL E O GERENCIAMENTO DE RESULTADOS CONTÁBEIS}

Desai e Dharmapala (2006) estimam que a gestão de resultados é mensurada pelos resíduos ou erros da regressão da conformidade Book-Tax em relação aos accruals. Pesquisas de Frank, Lynch, e Rego (2009) sinalizam na mesma direção, em que se desenvolve uma proxy de agressividade tributária, utilizando os resíduos ou erros da regressão da conformidade Book-Tax em relação aos accruals.

Um tema comum na literatura contábil sobre BTD é que estas estão associadas ao gerenciamento de resultados. Estudos de Lev e Nissim (2004), Plesko (2007), Bardertscher, Phillips, Pincus, e Rego (2009) evidenciam uma forte associação entre os componentes BTD com empresas com maior gerenciamento de resultados.

A relação entre a conformity Book-Tax e o earnings management aplica um modelo econométrico, que segue:

$$
E M_{i, t}=\beta_{0}+\beta_{1} \text { BTax }_{i t}+\beta_{2} S I Z E_{i t}+\beta_{3} L E V_{i t}+\beta_{4} R O A_{i t}+\beta_{5} G R O W T H_{i t}+\varepsilon_{i t}
$$

Em que:

$E M_{i t}=$ Erro do gerenciamento de resultados do modelo 2,

$\mathrm{BTax}_{\text {it }}=$ Erro da conformidade Book-Tax do modelo 1,

$\mathrm{SIZE}_{\text {it }}=\log$ Ativos Totais,

$\mathrm{LEV}_{\text {it }}=$ Passivo não circulante/Patrimônio Líquido,

$\mathrm{ROA}_{\text {it }}=$ Retorno sobre ativos,

Growth $_{i t}=$ Crescimento das Vendas.

Na seção seguinte os resultados são expostos e analisados. 


\section{RESULTADOS}

\subsection{MODELO 1 - CONFORMIDADE CONTÁBIL-FISCAL}

Nesse modelo, a variável dependente analisada é a CTE (despesas de IRPJ e CSSL corrente) e as variáveis independentes são PTBI (LAIR) e DIV (dividendos).

Uma estatística descritiva: média, desvio padrão e total de casos utilizados na modelagem estão apresentados em valores absolutos, a seguir, na Tabela 2:

Tabela 2

Estatística descritiva

\begin{tabular}{lrrr}
\hline & $\mathrm{N}$ & Média & \multicolumn{1}{c}{ Desvio padrão } \\
\hline CTE & 2452 & $139.562,34$ & $1.142 .962,84$ \\
PTBI & 2452 & $405.431,65$ & $4.425 .288,87$ \\
DIV & 2452 & $4.647,04$ & $25.458,87$ \\
\hline
\end{tabular}

Os resultados mostram que o modelo proposto é válido ( $p$-valor $=0,000$ ). O R2ajustado encontrado é de $76,2 \%$. Isso significa que $76,2 \%$ da variação presente no CTE são explicadas pelo PTBI e DIV, conforme explicado na Tabela 3 . Os $23,8 \%$ da variação são atribuídas ou a um efeito aleatório ou a outras variáveis não testadas.

Tabela 3

Sumário do modelo

\begin{tabular}{cccc}
\hline $\mathrm{R}$ & $\mathrm{R} \mathrm{2}$ & $\mathrm{R} 2$ ajustado & Sig. \\
\hline, $873^{\mathrm{a}}$ &, 763 &, 762 &, 000 \\
\hline
\end{tabular}

De acordo com Atwood et al. (2010), a conformidade contábil-fiscal das empresas tem origem na variação das despesas tributárias correntes com resultados positivos.

\subsection{MODELO 2 - GERENCIAMENTO DE RESULTADOS CONTÁBEIS}

No segundo modelo, a variável dependente analisada é a TACC (Accruals totais) e as variáveis independentes são, quando possível, escalonadas pelos ativos totais, usadas como 1/A $\mathrm{it}^{\prime}$ REV-AR (Variação da receita - contas a receber), PPE (Ativo Imobilizado +Ativo Intangível) e ROA (Retorno dos Ativos). 
Tabela 4

Coeficientes do modelo 2

\begin{tabular}{|c|c|c|c|c|}
\hline & \multicolumn{2}{|c|}{ Coeficientes } & \multirow{2}{*}{$t$} & \multirow{2}{*}{ Sig. } \\
\hline & $B$ & Erro Padrão & & \\
\hline (Constante) & -,017 & , 027 &,- 644 &, 520 \\
\hline $1 / A_{i t}$ & $-40,996$ & 5,639 & $-7,270$ & ,000 \\
\hline VRAR & ,000 &, 000 & 145 & 885 \\
\hline PPE &, 000 & ,000 & - 156 & 876 \\
\hline ROA &,- 002 & ,000 & $-4,263$ & ,000 \\
\hline
\end{tabular}

Nota. Significância do modelo $=0,000$. Variáveis significativas em negrito (Sig).

Ao gerar o modelo se observou que apenas as variáveis $1 / A_{i t}$ e ROA são significativas, ou seja, explicam TACC. A Tabela 4 mostra os coeficientes do modelo ajustado. É possível observar que a influência de $1 / A_{i t}$ e ROA é negativa, ou seja, seu aumento causa uma queda no TACC. O Erro gerado nesse modelo será chamado de Accruals discricionário (EM Earning Management) e será utilizado no modelo 3.

Para Beneish (2001, p. 3), estudos que utilizam os accruals totais para medir o gerenciamento de resultados são comuns na literatura e isso ocorre por causa dos seguintes fatores: a origem dos accruals, que surgem da diferença temporal entre os fluxos de caixa gerados pelas transações e os efeitos econômicos; dificuldades de mensuração dos efeitos das escolhas contábeis nos resultados; dificuldades dos usuários da informação contábil em detectar o gerenciamento de resultados nos números reportados.

\subsection{MODELO 3 - TESTE DA RELAÇÃO ENTRE A CONFORMIDADE CONTÁBIL-FISCAL E O GERENCIAMENTO DE RESULTADOS}

O modelo 3 tem como variável dependente o accruals discricionário (EM - Earning Management), que foi obtida por meio do modelo 2.

As variáveis independentes de interesse são: BTaxC (Conformidade Contábil-Fiscal) coletada no modelo 1, SIZE, LEV, ROA e GROWTH. Esse conjunto de variáveis explica 12,9\% da variação presente no $E M$.

Com esse conjunto, o modelo não foi significativo, conforme descrito na Tabela 5, ou seja, não existe relação entre Earnings Management (EM) e nenhuma das variáveis listadas.

Do conjunto de variáveis apresentadas, três delas são significativas (nesse caso utilizando 10\% de significância): SIZE (valor-p =0,066), ROA (valor-p =0,000) e BTaxC (valor-p $=0,012$ ). No entanto, os valores dos coeficientes de influência sobre EM são muito pequenos, mas positivos, ou seja, um aumento nessas variáveis acarreta um aumento em EM. 
Tabela 5

Coeficientes do modelo

\begin{tabular}{|c|c|c|c|c|c|c|}
\hline & \multicolumn{2}{|c|}{ Coeficientes } & \multirow[b]{2}{*}{$t$} & \multirow[b]{2}{*}{ Sig. } & \multicolumn{2}{|c|}{$95 \%$ Intervalo de confiança para B } \\
\hline & B & Erro padrão & & & Limite inferior & Limite superior \\
\hline (Constant) & 018 &, 030 & 600 &, 549 &,- 041 & 077 \\
\hline SIZE & ,00049 & ,000 & 1,843 & 066 & ,000 & , 000 \\
\hline LEV & ,00034 & ,002 & 169 & 866 &,- 004 & ,004 \\
\hline ROA & 0019 & 000 & 6,382 & 000 & ,001 & ,003 \\
\hline GROWTH &,- 00013 & ,000 &,- 351 & ,726 & ,000 & , 000 \\
\hline BTaxC &,- 00016 & ,000 & $-2,531$ & 012 & ,000 & ,000 \\
\hline $\mathrm{R}$ & R 2 & R 2 ajustado & Erro $\mathrm{p}$ & rão & Valor $\mathrm{p} \mathrm{d}$ & modelo \\
\hline 377 & 142 & 129 & & 37859859, & & , 000 \\
\hline
\end{tabular}

Com um valor de R2 sendo muito baixo (12,9\%), o resultado de EM será utilizado com seus valores absolutos na tentativa de identificar posições onde se possam oferecer respostas mais seguras à relação entre conformidade Book-Tax e o gerenciamento de resultados contábeis. Essa análise está na sequência.

\subsection{MODELO USANDO O ERRO 2 (EM) EM VALORES ABSOLUTOS - GERENCIAMENTO DE RESULTADOS CONTÁBEIS}

Quando se trabalha com o valor do erro, em formato absoluto, apresenta-se um resultado mais bem ajustado, em que o modelo passa de $12,9 \%$ de explicação para $33,3 \%$, conforme mostra a Tabela 6:

Tabela 6

Sumário do modelo

\begin{tabular}{rrrrr}
\hline $\mathrm{R}$ & $\mathrm{R} 2$ & \multicolumn{2}{c}{$\mathrm{R} 2$ ajustado } & \multicolumn{2}{c}{ Erro padrão } & \multicolumn{2}{c}{ Valor p do modelo } \\
\hline ,586 &, 343 &, 333 &, 3069 &, 000 \\
\hline
\end{tabular}

Alguns sinais se inverteram em relação ao modelo inicial: SIZE era positivo e ficou negativo; LEV e ROA eram positivos e passaram a negativos; $B$ Tax $C$ era negativo e ficou positivo. Essas três variáveis são as únicas significativas ao nível de $5 \%$ (SIZE = 0,021; ROA $=0,000$ e $B$ Tax $C=0,005)$, conforme a Tabela 7 : 
Tabela 7

Coeficientes do modelo

\begin{tabular}{|c|c|c|c|c|c|c|}
\hline & \multicolumn{4}{|c|}{$\begin{array}{c}\text { Coefficientes não } \\
\text { padronizados }\end{array}$} & \multicolumn{2}{|c|}{$\begin{array}{l}\text { 95\% Intervalo de Confiança } \\
\text { for B }\end{array}$} \\
\hline & B & Std. Error & $t$ & Sig. & Limite inferior & $\begin{array}{l}\text { Limite } \\
\text { superior }\end{array}$ \\
\hline (Constant) &, 075 & ,024 & 3,076 & ,002 & ,027 & , 123 \\
\hline SIZE & ,00050 & ,000 & $-2,319$ & ,021 & ,000 & ,000 \\
\hline LEV &,- 00020 &, 002 &,- 124 & 901 &,- 003 &, 003 \\
\hline ROA & -0029 & ,000 & $-11,932$ & ,000 &,- 003 &,- 002 \\
\hline GROWTH &,- 00025 &, 000 &,- 803 &, 423 &, 000 & ,000 \\
\hline BTaxC & ,00015 &, 000 & 2,860 &, 005 & ,000 & ,000 \\
\hline
\end{tabular}

Nota. Variáveis significativas em negrito (Sig).

Os resultados indicam quanto maior o $\mathrm{BTaxC}$, ou seja, quanto menor a conformidade contábil-fiscal, maior será a propensão ao gerenciamento de resultados. Em outras palavras, quanto menor fosse o BTaxC (mais conformidade contábil-fiscal), menor seria a propensão ao gerenciamento de resultados.

A conformidade contábil-fiscal inibe o gerenciamento de resultados contábeis, na medida em que o ônus tributário constitui um custo significativo para as empresas e estas preferem não gerenciar os resultados a ter que incorrer em carga tributária maior. Entretanto, quando existe uma baixa conformidade contábil-fiscal, estas podem gerenciar os seus resultados contábeis sem incorrer no ônus tributário de pagar tributos mais expressivos. De certo modo, a baixa conformidade contábil-fiscal constitui um incentivo econômico ao gerenciamento de resultados, pois diminui o custo tributário esperado dessas práticas.

Segundo Hanlon (2005), uma baixa conformidade contábil-fiscal provoca mudanças na avaliação do desempenho financeiro da companhia por parte dos investidores $e$ os lucros reportados são menos persistentes. Para Desai (2005), uma baixa conformidade contábil-fiscal reduz a qualidade do lucro divulgado, sendo um indicativo de gerenciamento de resultado pelos gestores. Estudos de Heltzer (2009) apontam que empresas com baixa conformidade contábil-fiscal, decorrente de gerenciamentos de resultados e agressividade fiscal, estão associadas com um baixo conservadorismo contábil.

Os resultados detectados no Brasil no período pós adoção do IFRS reforçam o que já tinha sido documentado internacionalmente, de que a ruptura da conformidade contábilfiscal e o consequente distanciamento do percurso entre o lucro contábil e o lucro fiscal contribuem para que se tenha uma contabilidade com menor qualidade em termos de accruals discricionários. Portanto, quanto maior forem as diferenças entre as regras contábeis e fiscais de um setor ou empresas para apurar o lucro contábil e fiscal, redobrada deve ser a atenção para eventuais práticas de gerenciamento de resultados. 
Ainda na Tabela 7 se demonstra que à medida que o tamanho da empresa e o ROA aumentam, o $E M$ diminui. $O$ efeito provocado pelo ROA é maior, pois seu coeficiente $(-0,0029)$ é maior que o coeficiente do tamanho da empresa $(-0,00050)$, e isso causa um impacto maior no $E M$, nesse caso, um impacto em direção à redução do $E M$.

Após a análise efetuada, cabe agora apresentar as conclusões.

\section{CONCLUSÕES}

Esta pesquisa buscou examinar se a conformidade contábil-fiscal pode restringir a divulgação oportunista dos resultados contábeis pelos gestores das empresas brasileiras de capital aberto, listadas na B3 - Brasil, Bolsa, Balcão, no período de 2010 a 2016. Os modelos implementados foram testados a partir de uma análise de regressões múltiplas.

De acordo com a literatura contábil, o desajuste entre o lucro contábil e o lucro tributável denominado BTD - Book-Tax Difference tem sua origem nas seguintes situações: de natureza discricionária, isto é, resultante de comportamentos oportunísticos dos gestores, e das diferenças entre as regras contábeis e as regras tributáveis.

Uma medida alternativa de conformidade contábil-fiscal foi desenvolvida por Atwood et al. (2010), sendo construída a partir de informações financeiras disponíveis. $O$ modelo econométrico para analisar o gerenciamento de resultado foi utilizado, bem como o modelo de Dechow et al. (1995), conhecido como Modelo Jones Modificado, ajustado pelo desempenho como proposto por Kothari et al. (2005). E um terceiro modelo econométrico foi utilizado para análise do teste da relação entre a conformidade Book-Tax e o gerenciamento de resultados.

Os resultados mostraram que quanto menor a conformidade contábil-fiscal, maior é a propensão ao gerenciamento de resultados. Adicionalmente, foi identificado que as empresas de menor tamanho e com menor rentabilidade tendem a gerenciar mais seus resultados contábeis. Os achados indicam que práticas contábeis que reduzem a conformidade das regras da contabilidade societária com aquelas das normas fiscais estimulam o aumento do gerenciamento de resultados contábeis. Por sua vez, naquelas empresas com maior conformidade contábil-fiscal há uma menor propensão a práticas de gerenciamento de resultados contábeis. O grau de conformidade contábil-fiscal passa a ser, assim, um elemento determinante para compreender os incentivos ao gerenciamento de resultados contábeis no Brasil.

Face às conclusões expostas, os resultados aqui documentados são de particular interesse para reguladores, preparadores de demonstrações financeiras e em especial usuários, que tomam decisões com base nas demonstrações contábeis publicadas no Brasil. É crucial notarem-se os incentivos econômicos gerados pela baixa conformidade contábil-fiscal 
para as práticas de gerenciamento de resultados e, diante disso, estar alerta a esse aspecto na avaliação da qualidade dos lucros contábeis.

Acrescenta-se que estudos anteriores a Mills e Newberry (2001) constataram igualmente que empresas com baixa conformidade contábil-fiscal têm maior probabilidades e incentivos econômicos para realizar o gerenciamento de resultados. Entretanto, o trabalho é de suma relevância por focar na realidade brasileira e apreciar justamente um período após a adoção das normas contábeis internacionais (IFRS). Se por um lado as normas internacionais introduzem um padrão informativo de maior relevância para os diversos usuários, elas criam regras que muitas vezes se afastam daquelas aplicadas para fins fiscais. Nesse cenário tem-se a baixa conformidade contábil-fiscal como um trade-off decorrente da introdução das normas internacionais.

Deve-se reconhecer que a pesquisa apresenta algumas limitações:

a) não foi investigada a relação da conformidade contábil-fiscal com práticas de gerenciamento dos resultados por decisões operacionais, que podem também interagir com aquelas baseadas em gerenciamento de resultados por accruals. As motivações para o gerenciamento de resultados por accruals e aquele por decisões operacionais podem ser distintas em face da conformidade fiscal, justificando análises adicionais;

b) a amostra é composta, particularmente, por empresas listadas em Bolsa e não levou em consideração as empresas fechadas, que podem ter outros incentivos econômicos, portanto, as conclusões aqui relatadas não teriam aplicação para elas. Nas empresas fechadas, a preocupação em atender às expectativas do mercado tem uma relevância expressivamente menor.

Como potencial expansão da pesquisa, caberia explorar as limitações apontadas, sendo recomendável uma discussão envolvendo a relação entre a conformidade contábilfiscal e o gerenciamento de resultados por accruals e por decisões operacionais, bem como uma aplicação de novos modelos econométricos em extensão de um maior espaço temporal de análise, e, se possível, incluindo empresas abertas e fechadas para uma visão mais abrangente dos incentivos econômicos que podem permear a relação entre a conformidade contábil-fiscal e o gerenciamento de resultados. Testes adicionais poderiam envolver a utilização de outros modelos para estimar a conformidade contábil-fiscal e os accruals discricionários. 


\section{REFERÊNCIAS}

Atwood, T. J., Drake, M. S., \& Myers, L. A. (2010). Book-tax conformity, earnings persistence and the association between earnings and future cash flows. Journal of Accounting and Economics, 50(1), 111-125.

Bardertscher, B., Phillips, J., Pincus, M., \& Rego, S. (2009). Earnings management strategies and the trade-off between tax benefits and detection risk: To conform or not to conform. The Accounting Review, 84(1), 63-97.

Beneish, M. D. (2001). Earnings management: A perspective. Managerial Finance. West Yorkshire, 27(12), 3-18.

Blaylock, B., Gaertner, F., \& Shevlin, T. (2015).The association between book-tax conformity and earnings management. Review of Accounting Studies, 20(1), 141-172.

Blaylock, B., Shevlin, T., \& Wilson, R. J. (2012). Tax avoidance, large positive temporary booktax differences, and earnings persistence. The Accounting Review, 87(1), 91-120.

Burgstaler, D., Leuz, C., \& Hail, L. (2006). The importance of reporting incentives: Earnings management in European private and public firms. The Accounting Review, 81, 983-1016.

Chan, K. H., Lin, K. Z., \& Tang, F. (2013). Tax Effects of Book-Tax Conformity, Financial reporting incentives, and firm size. Journal of International Accounting Research, 12(2), $1-25$.

Comprix, J., Graham, R. C., \& Moore, J. A. (2011). Empirical evidence on the impact of booktax differences on divergence of opinion among investors. Journal of the American Taxation Association, 33(1), 57-78.

Dechow, P. M., \& Dichev, I. D. (2002). The quality of accruals and earnings: The role of accruals estimation errors. The Accounting Review, 77(4), 35-59.

Dechow, P. M., Sloan, R. G., \& Sweney, A. P. (1995, April). Detecting earnings management. The Accounting Review, 70(2), 193-225.

Desai, M. A., \& Dharmapala, D. (2006). Corporate tax avoidance and high-powered incentives. Journal of Financial Economics, 79(1), 145-179.

Desai, M. A. (2005). The Degradation of Reported Corporate Profits. Journal of Economic Perspectives, 19(4), 171-192. 
Ferreira, F. R., Martinez, A. L., Costa, F. M., \& Passamani, R. R. (2012). Book-Tax Differences e gerenciamento de resultados no mercado de ações do Brasil. RAE - Revista de Administração de Empresas, 52(5).

Formigoni, H., Antunes, M. T., \& Paulo, E. (2009) Diferença entre o lucro contábil e lucro tributável: Uma análise sobre o gerenciamento de resultados contábeis e gerenciamento tributário nas companhias abertas brasileiras. BBR Brazilian Business Review, 6(1), 44-61.

Frank, M. M., Lynch, L. J., \& Rego, S. O. (2009). Tax reporting aggressiveness and its relation to aggressive financial reporting. The Accounting Review, 84(2), 467-469.

Goulart, A. M. C. (2007). Gerenciamento de resultados contábeis em instituições financeiras no Brasil. (Tese de doutorado). Universidade de São Paulo, São Paulo.

Graham, J. R., Raedy, J. S., \& Shackelford, D. A. (2012, February/April). Research in accounting for income taxes. Journal of Accounting and Economics, 53(1), 12-434.

Guenther, D. (2001). What do We Learn from Large Book Tax Difference? Paper presented at the meeting of University of Oregon, Eugene.

Hanlon, M., \& Heitzman, S. (2010). A review of tax research. Journal of Accounting and Economics, 50(2-3), 127-178.

Hanlon, M., \& Shevlin, H. T. (2005). Book-tax conformity for corporate income: An introduction to the issues. Tax Policy and the Economy, 19(1), 101-134.

Hanlon, M., Maydew, E. L., \& Shevlin, T. (2008). An unintended consequence of book-tax conformity: A loss of earnings in formativeness. Journal of Accounting and Economics, 46(2-3), 294-311.

Hanlon, M. (2005). The persistence and pricing of earnings, accruals and cash flows when firms have large book-tax differences. The Accounting Review, 80, 116-137.

Heltzer, W. (2009). Conservatism and book-tax differences. Journal of Accounting, Auditing \& Finance, 24(3), 469-504.

Kothari, S. P., Leone, A. J., \& Wasley, C. E. (2005). Performance matched discretionary accruals measures. Journal of Accounting and Economics, 39(1), 163-97.

Lei n. 11.638. (2007). Retirado de http://www.planalto.gov.br/ccivil_03/_ato2007-2010/2007/ lei/l11638.htm 
Lei n. 11.941. (2009). Retirado de http://www.planalto.gov.br/ccivil_03/_ato2007-2010/2009/ lei//11941.htm

Leuz, C., Nanda, D., \& Wysocki, P. D. (2003). Earnings management and investor protection: An international comparison. Journal of Financial Economics, 69, 505-527.

Lev, B., \& Nissim, D. (2004). Taxable income, future earnings, and equity values. The Accounting Review, 79(4), 1039-1074.

Martinez, A. L. (2001). Gerenciamento dos resultados contábeis: estudo empírico das companhias abertas brasileiras. (Tese de Doutorado). Universidade de São Paulo, São Paulo.

Martinez, A. L. (2008). Detectando earnings management no Brasil: Estimando os accruals discricionários. Revista de Contabilidade e Finanças-USP, 19(46), 7-17.

Martinez, A. L. (2013). Gerenciamento de resultados no Brasil: Um survey da literatura. Brazilian Business Review, 10(4), 1-31.

Mills, L., Newberry, K. (2001). The Influence of Tax and Nontax cost on Book-tax Reporting Difference: Public and Private Firms. The Journal of the American Taxation Association, 23(1), 1-19.

Paulo, E. (2007). Manipulação das informações contábeis: Uma análise teórica e empírica sobre os modelos operacionais de detecção de gerenciamento de resultados. (Tese de doutorado). Universidade de São Paulo, São Paulo.

Paulo, E., Martins, E., \& Corrar, L J. (2007) Detecção do gerenciamento de resultados pela análise do diferimento tributário. RAE Revista de Administração de Empresas, 47(1), 4659.

Peek, E., Palepu, K. G., \& Healy P. (2010) Business analysis and valuation. (2nd ed.). Boston: Cengage Learning.

Phillips, J., Pincus, M., \& Rego, S. (2003). Earnings management: New evidence based on deferred tax expense. The Accounting Review, 78, 491-52.

Plesko, G. (2007, May). Estimate of Magnitude of Financial and Tax Reporting Conflicts. Paper presented at the meeting of National Bureau of Economics Research, Cambridge. 
Riguen, K. R., \& Jarboui, A. (2017). Normal, abnormal book-tax differences and accounting conservatism. Asian Academy of Management. Journal of Accounting and Finance, 13(1), $113-142$.

Roychowdhury, S. (2006). Earnings management through realactivities manipulation. Journal of Accounting and Economics, 42(3), 335-370.

Tang, T. Y. H. (2014). Does Book-Tax conformity deter opportunistic book and tax reporting? An international analysis. European Accounting Review, 24(3), 441-469.

Tang, T. Y. H., \& Firth, M. (2012). Earnings persistence and stock market reactions to the different information in book-tax differences: Evidence from China. The International Journal of Accounting, 47(3), 369-397.

Wahab, N. S. A., \& Holland, K. (2015). The persistence of book-tax differences. The British Accounting Review, 47(4), 339-350.

\section{Como citar este artigo:}

\section{ABNT}

MARTINEZ, Antonio Lopo; LEAL, Lennilton Viana. Conformidade contábil-fiscal e gerenciamento de resultados contábeis no Brasil. RACE, Revista de Administração, Contabilidade e Economia, Joaçaba: Ed. Unoesc, v. 18, n. 1, p. 9-30, jan./abr. 2019. Disponível em: https://portalperiodicos.unoesc.edu.br/race. Acesso em: dia/mês/ano.

\section{APA}

Martinez, A. L., \& Leal, L. V. (2019). Conformidade contábil-fiscal e gerenciamento de resultados contábeis no Brasil. RACE, Revista de Administração, Contabilidade e Economia, 18(1), 9-30. Recuperado de http://editora.unoesc.edu.br/index.php/race 
